\title{
Kalman Filtering With Intermittent Observations
}

\author{
Bruno Sinopoli, Student Member, IEEE, Luca Schenato, Member, IEEE, Massimo Franceschetti, Member, IEEE, \\ Kameshwar Poolla, Member, IEEE, Michael I. Jordan, Senior Member, IEEE, and Shankar S. Sastry, Fellow, IEEE
}

\begin{abstract}
Motivated by navigation and tracking applications within sensor networks, we consider the problem of performing Kalman filtering with intermittent observations. When data travel along unreliable communication channels in a large, wireless, multihop sensor network, the effect of communication delays and loss of information in the control loop cannot be neglected. We address this problem starting from the discrete Kalman filtering formulation, and modeling the arrival of the observation as a random process. We study the statistical convergence properties of the estimation error covariance, showing the existence of a critical value for the arrival rate of the observations, beyond which a transition to an unbounded state error covariance occurs. We also give upper and lower bounds on this expected state error covariance.
\end{abstract}

Index Terms-Kalman estimation, missing observation, online adaptive filtering, sensor networks, stability.

\section{INTRODUCTION}

A DVANCES in very large-scale integration and microelectromechanical system technology have boosted the development of micro sensor integrated systems. Such systems combine computing, storage, radio technology, and energy source on a single chip [1], [2]. When distributed over a wide area, networks of sensors can perform a variety of tasks that range from environmental monitoring and military surveillance, to navigation and control of a moving vehicle [3]-[5]. A common feature of these systems is the presence of significant communication delays and data loss across the network. From the point of view of control theory, significant delay is equivalent to loss, as data needs to arrive to its destination in time to be used for control. In short, communication and control become tightly coupled such that the two issues cannot be addressed independently.

Consider, for example, the problem of navigating a vehicle based on the estimate from a sensor web of its current position and velocity. The measurements underlying this estimate can be lost or delayed due to the unreliability of the wireless links. What is the amount of data loss that the control loop can tolerate to reliably perform the navigation task? Can communication protocols be designed to satisfy this constraint? At Berkeley, we have faced these kind of questions in building sensor networks for pursuit evasion games as part of the network of embedded systems technology (NEST) project [2]. Practical advances in

Manuscript received May 21, 2003; revised December 5, 2003 and March 8, 2003. Recommended by Guest Editors P. Antsaklis and J. Baillieul. This work was supported by the Defense Advanced Research Projects Agency under Grant F33615-01-C-1895.

The authors are with the Department of Electrical Engineering and Computer Sciences, the University of California, Berkeley, CA 94720 USA (e-mail: sinopoli@eecs.berkeley.edu).

Digital Object Identifier 10.1109/TAC.2004.834121

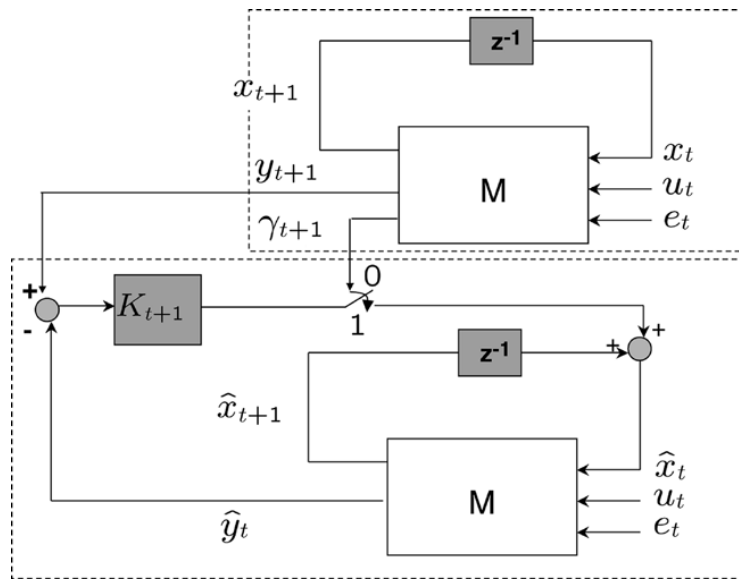

System

Fig. 1. Overview of the system. We study the statistical convergence of the expected estimation error covariance of the discrete Kalman filter, where the observation, travelling over an unreliable communication channel, can be lost at each time step with probability $1-\lambda$.

the design of these systems are described in [6]. The goal of this paper is to examine some control-theoretic implications of using sensor networks for control. These require a generalization of classical control techniques that explicitly take into account the stochastic nature of the communication channel.

In our setting, the sensor network provides observed data that are used to estimate the state of a controlled system, and this estimate is then used for control. We study the effect of data losses due to the unreliability of the network links. We generalize the most ubiquitous recursive estimation technique in control-the discrete-time Kalman filter [7] — modeling the arrival of an observation as a random process whose parameters are related to the characteristics of the communication channel, see Fig. 1. We characterize the statistical convergence of the expected estimation error covariance in this setting.

The classical theory relies on several assumptions that guarantee convergence of the Kalman filter. Consider the following discrete-time linear dynamical system:

$$
\begin{aligned}
x_{t+1} & =A x_{t}+w_{t} \\
y_{t} & =C x_{t}+v_{t}
\end{aligned}
$$

where $x_{t} \in \mathbb{R}^{n}$ is the state vector, $y_{t} \in \mathbb{R}^{m}$ the output vector, $w_{t} \in \mathbb{R}^{p}$ and $v_{t} \in \mathbb{R}^{m}$ are Gaussian random vectors with zero mean and covariance matrices $Q \geq 0$ and $R>0$, respectively. $w_{t}$ is independent of $w_{s}$ for $s<t$. Assume that the initial state, $x_{0}$, is also a Gaussian vector of zero mean and covariance $\Sigma_{0}$. Under the hypothesis of stabilizability of the pair $(A, Q)$ and detectability of the pair $(A, C)$, the estimation error covariance of the Kalman filter converges to a unique value from any initial condition [8]. 
These assumptions have been relaxed in various ways. Extended Kalman filtering [8] attempts to cope with nonlinearities in the model; particle filtering [9] is also appropriate for nonlinear models and additionally does not require the noise model to be Gaussian. Recently, more general observation processes have been studied. In particular, in [10] and [11], the case in which observations are randomly spaced in time according to a Poisson process has been studied, where the underlying dynamics evolve in continuous time. These authors showed the existence of a lower bound on the arrival rate of the observations below which it is possible to maintain the estimation error covariance below a fixed value, with high probability. However, the results were restricted to scalar single-input-single-output systems.

We approach a similar problem within the framework of discrete time, and provide results for general $n$-dimensional multiple-input-multiple-output (MIMO) systems. In particular, we consider a discrete-time system in which the arrival of an observation is a Bernoulli process with parameter $0<\lambda<1$, and, rather than asking for the estimation error covariance to be bounded with high probability, we study the asymptotic behavior (in time) of its average. Our main contribution is to show that, depending on the eigenvalues of the matrix $A$, and on the structure of the matrix $C$, there exists a critical value $\lambda_{c}$, such that if the probability of arrival of an observation at time $t$ is $\lambda>\lambda_{c}$, then the expectation of the estimation error covariance is always finite (provided that the usual stabilizability and detectability hypotheses are satisfied). If $\lambda \leq \lambda_{c}$, then the expectation of the estimation error covariance is unbounded. We give explicit upper and lower bounds on $\lambda_{c}$, and show that they are tight in some special cases.

Philosophically, this result can be seen as another manifestation of the well known uncertainty threshold principle [12], [13]. This principle states that optimum long-range control of a dynamical system with uncertainty parameters is possible if and only if the uncertainty does not exceed a given threshold. The uncertainty is modeled as white noise scalar sequences acting on the system and control matrices. In our case, the result is for optimal estimation, rather than optimal control, and the uncertainty is due to the random arrival of the observation, with the randomness arising from losses in the network.

Studies on filtering with intermittent observations can be tracked back to Nahi [14] and Hadidi [15]. More recently, this problem has been studied using jump linear systems (JLSs) [16]. JLSs are stochastic hybrid systems characterized by linear dynamics and discrete regime transitions modeled as Markov chains. In [17], [18], and [19], the Kalman filter with missing observations is modeled as a JLS switching between two discrete regimes: an open-loop configuration and a closed-loop one. Following this approach, these authors obtain convergence criteria for the expected estimation error covariance. However, they restrict their formulation to the steady-state case, where the Kalman gain is constant, and they do not assume to know the switching sequence. The resulting process is wide sense stationary [20], and this makes the exact computation of the transition probability and state error covariance possible. Other work on optimal, constant gain filtering can be found in the work of Wang et al. [21], who included the presence of system parameters uncertainty besides missing observations, and Smith et al. [22], who considered multiple filters fusion. Instead, we consider the general case of time varying Kalman gain. In presence of missing observations, this filter has a smaller linear minimum mean square error (LMMSE) than its static counterpart, as it is detailed in Section II.

The general case of time-varying Kalman filter with intermittent observations was also studied by Fortmann et al. [23], who derived stochastic equations for the state covariance error. However, they do not statistically characterize its convergence and provide only numerical evidence of the transition to instability, leaving a formal characterization of this as an open problem, which is addressed in this paper. A somewhat different formulation was considered in [24], where the observations arrival have a bounded delay.

Finally, we point out that our analysis can also be viewed as an instance of expectation-maximization (EM) theory. EM is a general framework for doing maximum likelihood estimation in missing-data models [25]. Lauritzen [26] shows how EM can be used for general graphical models. In our case, however, the graph structure is a function of the missing data, as there is one graph for each pattern of missing data.

The paper is organized as follows. In Section II, we formalize the problem of Kalman filtering with intermittent observations. In Section III, we provide upper and lower bounds on the expected estimation error covariance of the Kalman filter, and find the conditions on the observation arrival probability $\lambda$ for which the upper bound converges to a fixed point, and for which the lower bound diverges. Section IV describes some special cases and gives an intuitive understanding of the results. In Section V, we compare our approach to previous ones [18] based on jump linear systems. Finally, in Section VI, we state our conclusions and give directions for future work.

\section{PROBlem Formulation}

Consider the canonical state estimation problem. We define the arrival of the observation at time $t$ as a binary random variable $\gamma_{t}$, with probability distribution $p_{\gamma_{t}}(1)=\lambda_{t}$, and with $\gamma_{t}$ independent of $\gamma_{s}$ if $t \neq s$. The output noise $v_{t}$ is defined in the following way:

$$
p\left(v_{t} \mid \gamma_{t}\right)=\left\{\begin{array}{cc}
\mathcal{N}(0, R), & \gamma_{t}=1 \\
\mathcal{N}\left(0, \sigma^{2} I\right), & \gamma_{t}=0
\end{array}\right.
$$

for some $\sigma^{2}$. Therefore, the variance of the observation at time $t$ is $R$ if $\gamma_{t}$ is 1 , and $\sigma^{2} I$ otherwise. In reality the absence of observation corresponds to the limiting case of $\sigma \rightarrow \infty$. Our approach is to rederive the Kalman filter equations using a "dummy" observation with a given variance when the real observation does not arrive, and then take the limit as $\sigma \rightarrow \infty$.

First, let us define

$$
\begin{aligned}
\hat{x}_{t \mid t} & \triangleq \mathbb{E}\left[x_{t} \mid \mathbf{y}_{\mathbf{t}}, \gamma_{\mathbf{t}}\right] \\
P_{t \mid t} & \triangleq \mathbb{E}\left[\left(x_{t}-\hat{x}_{t}\right)\left(x_{t}-\hat{x}_{t}\right)^{\prime} \mid \mathbf{y}_{\mathbf{t}}, \gamma_{\mathbf{t}}\right] \\
\hat{x}_{t+1 \mid t} & \triangleq \mathbb{E}\left[x_{t+1} \mid \mathbf{y}_{\mathbf{t}}, \gamma_{\mathbf{t}}\right] \\
P_{t+1 \mid t} & \triangleq \mathbb{E}\left[\left(x_{t+1}-\hat{x}_{t+1}\right)\left(x_{t+1}-\hat{x}_{t+1}\right)^{\prime} \mid \mathbf{y}_{\mathbf{t}}, \gamma_{\mathbf{t}}\right] \\
\hat{y}_{t+1 \mid t} & \triangleq \mathbb{E}\left[y_{t+1} \mid \mathbf{y}_{\mathbf{t}}, \gamma_{\mathbf{t}}\right]
\end{aligned}
$$


where we have defined the vectors $\mathbf{y}_{\mathbf{t}} \triangleq\left[y_{0}, \ldots, y_{t}\right]^{\prime}$ and $\gamma_{\mathbf{t}} \triangleq$ $\left[\gamma_{0}, \ldots, \gamma_{t}\right]^{\prime}$. It is easy to see that

$$
\begin{aligned}
& \mathbb{E}\left[\left(y_{t+1}-\hat{y}_{t+1 \mid t}\right)\left(x_{t+1}-\hat{x}_{t+1 \mid t}\right)^{\prime} \mid \mathbf{y}_{\mathbf{t}}, \gamma_{\mathbf{t}+\mathbf{1}}\right] \\
& \quad=C P_{t+1 \mid t} \\
& \mathbb{E}\left[\left(y_{t+1}-\hat{y}_{t+1 \mid t}\right)\left(y_{t+1}-\hat{y}_{t+1 \mid t}\right)^{\prime} \mid \mathbf{y}_{\mathbf{t}}, \gamma_{\mathbf{t + 1}}\right] \\
& \quad=C P_{t+1 \mid t} C^{\prime}+\gamma_{t+1} R+\left(1-\gamma_{t+1}\right) \sigma^{2} I
\end{aligned}
$$

and it follows that the random variables $x_{t+1}$ and $y_{t+1}$, conditioned on the output $\mathbf{y}_{\mathbf{t}}$ and on the arrivals $\gamma_{\mathbf{t}+\mathbf{1}}$, are jointly Gaussian with mean

$$
\mathbb{E}\left[x_{t+1}, y_{t+1} \mid \mathbf{y}_{\mathbf{t}}, \gamma_{\mathbf{t + 1}}\right]=\left(\begin{array}{c}
\hat{x}_{t+1 \mid t} \\
C \hat{x}_{t+1 \mid t}
\end{array}\right)
$$

and covariance

$$
\begin{aligned}
\operatorname{COV} & \left(x_{t+1}, y_{t+1} \mid \mathbf{y}_{\mathbf{t}}, \gamma_{\mathbf{t}+\mathbf{1}}\right) \\
& =\left(\begin{array}{cc}
P_{t+1 \mid t} & P_{t+1 \mid t} C^{\prime} \\
C P_{t+1 \mid t} & C P_{t+1 \mid t} C^{\prime}+\gamma_{t+1} R+\left(1-\gamma_{t+1}\right) \sigma^{2} I
\end{array}\right) .
\end{aligned}
$$

Hence, the Kalman filter equations are modified as follows:

$$
\begin{aligned}
\hat{x}_{t+1 \mid t}= & A \hat{x}_{t \mid t} \\
P_{t+1 \mid t}= & A P_{t \mid t} A^{\prime}+Q \\
\hat{x}_{t+1 \mid t+1}= & \hat{x}_{t+1 \mid t}+P_{t+1 \mid t} C^{\prime} \\
& \times\left(C P_{t+1 \mid t} C^{\prime}+\gamma_{t+1} R+\left(1-\gamma_{t+1}\right) \sigma^{2} I\right)^{-1} \\
& \times\left(y_{t+1}-C \hat{x}_{t+1 \mid t}\right) \\
P_{t+1 \mid t+1}= & P_{t+1 \mid t}-P_{t+1 \mid t} C^{\prime} \\
& \times\left(C P_{t+1 \mid t} C^{\prime}+\gamma_{t+1} R+\left(1-\gamma_{t+1}\right) \sigma^{2} I\right)^{-1} \\
& \times C P_{t+1 \mid t} .
\end{aligned}
$$

Taking the limit as $\sigma \rightarrow \infty$, the update (11) and (12) can be rewritten as follows:

$$
\begin{aligned}
& \hat{x}_{t+1 \mid t+1}=\hat{x}_{t+1 \mid t}+\gamma_{t+1} K_{t+1}\left(y_{t+1}-C \hat{x}_{t+1 \mid t}\right) \\
& P_{t+1 \mid t+1}=P_{t+1 \mid t}-\gamma_{t+1} K_{t+1} C P_{t+1 \mid t}
\end{aligned}
$$

where $K_{t+1}=P_{t+1 \mid t} C^{\prime}\left(C P_{t+1 \mid t} C^{\prime}+R\right)^{-1}$ is the Kalman gain matrix for the standard ARE. Note that performing this limit corresponds exactly to propagating the previous state when there is no observation update available at time $t$. We also point out the main difference from the standard Kalman filter formulation: Both $\hat{x}_{t+1 \mid t+1}$ and $P_{t+1 \mid t+1}$ are now random variables, being a function of $\gamma_{t+1}$, which is itself random.

It is important to stress that (13) and (14) give the minimum state-error variance filter given the observations $\left\{y_{t}\right\}$ and their arrival sequence $\left\{\gamma_{t}\right\}$, i.e. $\hat{x}_{t}^{t m}=\mathbb{E}\left[x_{t} \mid y_{t}, \ldots, y_{1}, \gamma_{t}, \ldots, \gamma_{1}\right]$. As a consequence, the filter proposed in this paper is necessarily time-varying and stochastic since it depends on the arrival sequence. The filters that have been proposed so far using JLS theory [17], [19] give the minimum state error variance filters assuming that only the observations $\left\{y_{t}\right\}$ and the knowledge on the last arrival $\gamma_{t}$ are available, i.e. $\hat{x}_{t}^{J L S}=\mathbb{E}\left[x_{t} \mid y_{t}, \ldots, y_{1}, \gamma_{t}\right]$. Therefore, the filter given by (13) and (14) gives a better performance than its JLS counterparts, since it exploits additional information regarding the arrival sequence.

Given the new formulation, we now study the Riccati equation of the state error covariance matrix in the specific case when the arrival process of the observation is time-independent, i.e., $\lambda_{t}=\lambda$ for all time. This will allow us to provide deterministic upper and lower bounds on its expectation. We then characterize the convergence of these upper and lower bounds, as a function of the arrival probability $\lambda$ of the observation.

\section{Convergence Conditions And TRAnsition TO INSTABILITY}

It is easy to verify that the modified Kalman filter formulation in (10) and (14) can be rewritten as follows:

$$
P_{t+1}=A P_{t} A^{\prime}+Q-\gamma_{t} A P_{t} C^{\prime}\left(C P_{t} C^{\prime}+R\right)^{-1} C P_{t} A^{\prime}
$$

where we use the simplified notation $P_{t}=P_{t \mid t-1}$. Since the sequence $\left\{\gamma_{t}\right\}_{0}^{\infty}$ is random, the modified Kalman filter iteration is stochastic and cannot be determined offline. Therefore, only statistical properties can be deduced.

In this section, we show the existence of a critical value $\lambda_{c}$ for the arrival probability of the observation update, such that for $\lambda>\lambda_{c}$ the mean state covariance $\mathbb{E}\left[P_{t}\right]$ is bounded for all initial conditions, and for $\lambda \leq \lambda_{c}$ the mean state covariance diverges for some initial condition. We also find a lower bound $\underline{\lambda}$, and upper bound $\bar{\lambda}$, for the critical probability $\lambda_{c}$, i.e., $\underline{\lambda} \leq \lambda_{c} \leq \bar{\lambda}$. The lower bound is expressed in closed form; the upper bound is the solution of a linear matrix inequality (LMI). In some special cases the two bounds coincide, giving a tight estimate. Finally, we present numerical algorithms to compute a lower bound $\bar{S}$, and upper bound $\bar{V}$, for $\lim _{t \rightarrow \infty} \mathbb{E}\left[P_{t}\right]$, when it is bounded.

First, we define the modified algebraic Riccati equation (MARE) for the Kalman filter with intermittent observations as follows:

$$
g_{\lambda}(X)=A X A^{\prime}+Q-\lambda A X C^{\prime}\left(C X C^{\prime}+R\right)^{-1} C X A^{\prime} .
$$

Our results derive from two principal facts: The first is that concavity of the modified algebraic Riccati equation for our filter with intermittent observations allows use of Jensen's inequality to find an upper bound on the mean state covariance; the second is that all the operators we use to estimate upper and lower bounds are monotonically increasing, therefore, if a fixed point exists, it is also stable.

We formally state all main results in form of theorems. Omitted proofs appear in the Appendix. The first theorem expresses convergence properties of the MARE.

Theorem 1: Consider the operator $\phi(K, X)=(1-$ $\lambda)\left(A X A^{\prime}+Q\right)+\lambda\left(F X F^{\prime}+V\right)$, where $F=A+K C$, $V=Q+K R K^{\prime}$. Suppose there exists a matrix $\tilde{K}$ and a positive-definite matrix $\tilde{P}$ such that

$$
\tilde{P}>0 \quad \text { and } \quad \tilde{P}>\phi(\tilde{K}, \tilde{P}) .
$$

Then

a) for any initial condition $P_{0} \geq 0$, the MARE converges, and the limit is independent of the initial condition

$$
\lim _{t \rightarrow \infty} P_{t}=\lim _{t \rightarrow \infty} g_{\lambda}^{t}\left(P_{0}\right)=\bar{P}
$$

b) $\bar{P}$ is the unique positive-semidefinite fixed point of the MARE.

The next theorem states the existence of a sharp transition. 
Theorem 2: If $\left(A, Q^{1 / 2}\right)$ is controllable, $(A, C)$ is detectable, and $A$ is unstable, then there exists a $\lambda_{c} \in[0,1)$ such that

$$
\begin{aligned}
& \lim _{t \rightarrow \infty} \mathbb{E}\left[P_{t}\right]=+\infty, \quad \text { for } 0 \leq \lambda \leq \lambda_{c} \text { and } \exists P_{0} \geq 0 \\
& \mathbb{E}\left[P_{t}\right] \leq M_{P_{0}} \forall t, \quad \text { for } \lambda_{c}<\lambda \leq 1 \text { and } \forall P_{0} \geq 0
\end{aligned}
$$

where $M_{P_{0}}>0$ depends on the initial condition $P_{0} \geq 0 .{ }^{1}$

The next theorem gives upper and lower bounds for the critical probability $\lambda_{c}$.

Theorem 3: Let

$$
\begin{aligned}
& \underline{\lambda}=\operatorname{argin} f_{\lambda}\left[\exists \hat{S} \mid \hat{S}=(1-\lambda) A \hat{S} A^{\prime}+Q\right]=1-\frac{1}{\alpha^{2}} \\
& \bar{\lambda}=\operatorname{argin} f_{\lambda}[\exists(\hat{K}, \hat{X}) \mid \hat{X}>\phi(\hat{K}, \hat{X})]
\end{aligned}
$$

where $\alpha=\max _{i}\left|\sigma_{i}\right|$ and $\sigma_{i}$ are the eigenvalues of $A$. Then

$$
\underline{\lambda} \leq \lambda_{c} \leq \bar{\lambda}
$$

Finally, the following theorem gives an estimate of the limit of the mean covariance matrix $\mathbb{E}\left[P_{t}\right]$, when this is bounded.

Theorem 4: Assume that $\left(A, Q^{1 / 2}\right)$ is controllable, $(A, C)$ is detectable and $\lambda>\bar{\lambda}$, where $\bar{\lambda}$ is defined in Theorem 4 . Then

$$
0<S_{t} \leq \mathbb{E}\left[P_{t}\right] \leq V_{t} \quad \forall \mathbb{E}\left[P_{0}\right] \geq 0
$$

where $\lim _{t \rightarrow \infty} S_{t}=\bar{S}$ and $\lim _{t \rightarrow \infty} V_{t}=\bar{V}$, where $\bar{S}$ and $\bar{V}$ are solution of the respective algebraic equations $\bar{S}=(1-$ ג) $A \bar{S} A^{\prime}+Q$ and $\bar{V}=g_{\lambda}(\bar{V})$.

The previous theorems give lower and upper bounds for both the critical probability $\lambda_{c}$ and for the mean error covariance $\mathbb{E}\left[P_{t}\right]$. The lower bound $\underline{\lambda}$ is expressed in closed form. We now resort to numerical algorithms for the computation of the remaining bounds $\bar{\lambda}, \bar{S}$ and $\bar{V}$.

The computation of the upper bound $\bar{\lambda}$ can be reformulated as the iteration of an LMI feasibility problem. To establish this, we need the following theorem.

Theorem 5: If $\left(A, Q^{1 / 2}\right)$ is controllable and $(A, C)$ is detectable, then the following statements are equivalent.

a) $\exists \bar{X}$ such that $\bar{X}>g_{\lambda}(\bar{X})$.

b) $\exists \bar{K}, \bar{X}>0$ such that $\bar{X}>\phi(\bar{K}, \bar{X})$.

c) $\exists \bar{Z}$ and $0<\bar{Y} \leq I$ such that

$$
\begin{aligned}
\Psi_{\lambda}(Y, Z) & =\left[\begin{array}{ccc}
Y & \sqrt{\lambda}(Y A+Z C) & \sqrt{1-\lambda} Y A \\
\sqrt{\lambda}\left(A^{\prime} Y+C^{\prime} Z^{\prime}\right) & Y & 0 \\
\sqrt{1-\lambda} A^{\prime} Y & 0 & Y
\end{array}\right] \\
& >0
\end{aligned}
$$

Proof: a) $\Longrightarrow$ b) If $\bar{X}>g_{\lambda}(\bar{X})$ exists, then $\bar{X}>0$ by Lemma $1(\mathrm{~g})$. Let $\bar{K}=K_{\bar{X}}$. Then $\bar{X}>g_{\lambda}(\bar{X})=\phi(\bar{K}, \bar{X})$ which proves the statement.

b) $\Longrightarrow$ a) Clearly $\bar{X}>\phi(\bar{K}, \bar{X}) \geq g_{\lambda}(\bar{X})$ which proves the statement.

b) $\Longleftrightarrow$ c) Let $F=A+K C$, then

$$
X>(1-\lambda) A X A^{\prime}+\lambda F X F^{\prime}+Q+\lambda K R K^{\prime}
$$

${ }^{1}$ We use the notation $\lim _{t \rightarrow \infty} A_{t}=+\infty$ when the sequence $A_{t} \geq 0$ is not bounded, i.e., there is no matrix $M \geq 0$ such that $A_{t} \leq M, \forall t$. is equivalent to

$$
\left[\begin{array}{cc}
X-(1-\lambda) A X A^{\prime} & \sqrt{\lambda} F \\
\sqrt{\lambda} F^{\prime} & X^{-1}
\end{array}\right]>0
$$

where we used the Schur complement decomposition and the fact that $X-(1-\lambda) A X A^{\prime} \geq \lambda F X F^{\prime}+Q+\lambda K R K^{\prime} \geq Q>0$. Using one more time the Schur complement decomposition on the first element of the matrix we obtain

$$
\Theta=\left[\begin{array}{ccc}
X & \sqrt{\lambda} F & \sqrt{1-\lambda} A \\
\sqrt{\lambda} F^{\prime} & X^{-1} & 0 \\
\sqrt{1-\lambda} A^{\prime} & 0 & X^{-1}
\end{array}\right]>0 .
$$

This is equivalent to

$$
\begin{aligned}
\Lambda & =\left[\begin{array}{ccc}
X^{-1} & 0 & 0 \\
0 & I & 0 \\
0 & 0 & I
\end{array}\right] \Theta\left[\begin{array}{ccc}
X^{-1} & 0 & 0 \\
0 & I & 0 \\
0 & 0 & I
\end{array}\right]>0 \\
& =\left[\begin{array}{ccc}
X^{-1} & \sqrt{\lambda} X^{-1} F & \sqrt{1-\lambda} X^{-1} A \\
\sqrt{\lambda} F^{\prime} X^{-1} & X^{-1} & 0 \\
\sqrt{1-\lambda} A^{\prime} X^{-1} & 0 & X^{-1}
\end{array}\right]>0 .
\end{aligned}
$$

Let us consider the change of variable $Y=X^{-1}>0$ and $Z=X^{-1} K$, in which case the previous LMI is equivalent to

$$
\begin{aligned}
\Psi(Y, Z) & =\left[\begin{array}{ccc}
Y & \sqrt{\lambda}(Y A+Z C) & \sqrt{1-\lambda} Y A \\
\sqrt{\lambda}\left(A^{\prime} Y+C^{\prime} Z^{\prime}\right) & Y & 0 \\
\sqrt{1-\lambda} A^{\prime} Y & 0 & Y
\end{array}\right] \\
& >0 .
\end{aligned}
$$

Since $\Psi(\alpha Y, \alpha K)=\alpha \Psi(Y, K)$, then $Y$ can be restricted to $Y \leq I$, which completes the theorem.

Combining Theorems 3 and 5, we immediately have the following corollary.

Corollary 1: The upper bound $\bar{\lambda}$ is given by the solution of the following optimization problem:

$$
\bar{\lambda}=\operatorname{argmin}_{\lambda} \Psi_{\lambda}(Y, Z)>0, \quad 0 \leq Y \leq I .
$$

This is a quasi-convex optimization problem in the variables $(\lambda, Y, Z)$ and the solution can be obtained by iterating LMI feasibility problems and using bisection for the variable $\lambda$, as shown in [27].

The lower bound $\bar{S}$ for the mean covariance matrix can be easily obtained via standard Lyapunov Equation solvers. The upper bound $\bar{V}$ can be found by iterating the MARE or by solving a semidefinite programming (SDP) problem as shown in the following.

Theorem 6: If $\lambda>\bar{\lambda}$, then the matrix $\bar{V}=g_{\lambda}(\bar{V})$ is given by

a) $\bar{V}=\lim _{t \rightarrow \infty} V_{t} ; V_{t+1}=g_{\lambda}\left(V_{t}\right)$ where $V_{0} \geq 0$

b)

$\begin{array}{lc}\operatorname{argmax}_{V} & \operatorname{Trace}(V) \\ \text { subject to } & {\left[\begin{array}{cc}A V A^{\prime}-V & \sqrt{\lambda} A V C^{\prime} \\ \sqrt{\lambda} C V A^{\prime} & C V C^{\prime}+R\end{array}\right] \geq 0, \quad V \geq 0 .}\end{array}$

Proof:

a) It follows directly from Theorem 1 . 


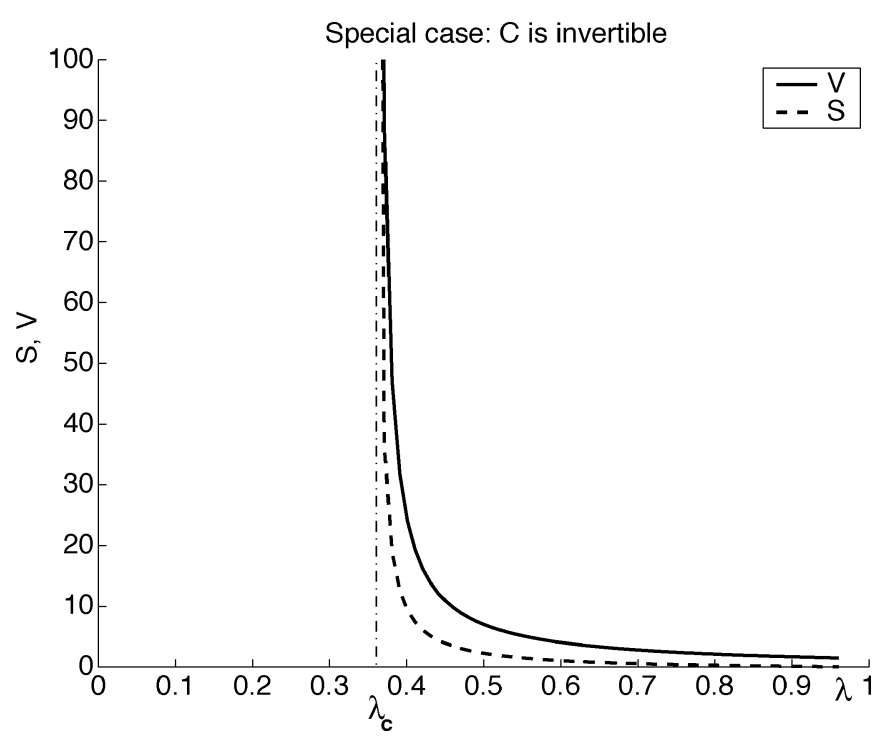

Fig. 2. Example of transition to instability in the scalar case. The dashed line shows the asymptotic value of the lower bound $(\bar{S})$, the solid line the asymptotic value of the upper bound $(\bar{V})$, and the dashed-dotted line shows the asymptote $\left(\lambda_{c}\right)$.

b) It can be obtained by using the Schur complement decomposition on the equation $V \leq g_{\lambda}(V)$. Clearly, the solution $\bar{V}=g_{\lambda}(\bar{V})$ belongs to the feasible set of the optimization problem. We now show that the solution of the optimization problem is the fixed point of the MARE. Suppose it is not, i.e., $\hat{V}$ solves the optimization problem but $\hat{V} \neq g_{\lambda}(\hat{V})$. Since $\hat{V}$ is a feasible point of the optimization problem, then $\hat{V}<g_{\lambda}(\hat{V})=\hat{\hat{V}}$. However, this implies that $\operatorname{Trace}(\hat{V})<\operatorname{Trace}(\hat{\hat{V}})$, which contradicts the hypothesis of optimality of matrix $\hat{V}$. Therefore, $\hat{V}=g_{\lambda}(\hat{V})$ and this concludes the theorem.

\section{SPecial CASES AND EXAMPLES}

In this section, we present some special cases in which upper and lower bounds on the critical value $\lambda_{c}$ coincide and give some examples. From Theorem 1, it follows that if there exists a $\tilde{K}$ such that $F$ is the zero matrix, then the convergence condition of the MARE is for $\lambda>\lambda_{c}=1-1 / \alpha^{2}$, where $\alpha=\max _{i}\left|\sigma_{i}\right|$, and $\sigma_{i}$ are the eigenvalues of $A$.

- $\mathbf{C}$ is invertible: In this case, a choice of $K=-A C^{-1}$ makes $F=0$. Note that the scalar case also falls under this category. Fig. 2 shows a plot of the steady state of the upper and lower bounds versus $\lambda$ in the scalar case. The discrete time LTI system used in this simulation has $A=-1.25$, $C=1$, with $v_{t}$ and $w_{t}$ having zero mean and variance $R=2.5$ and $Q=1$, respectively. For this system, we have $\lambda_{c}=0.36$. The transition clearly appears in the figure, where we see that the steady-state value of both upper and lower bound tends to infinity as $\lambda$ approaches $\lambda_{c}$. The dashed line shows the lower bound, the solid line the upper bound, and the dashed-dotted line shows the asymptote.

- A has a single unstable eigenvalue: In this case, regardless of the dimension of $C$ (and as long as the pair $(A, C)$ is detectable), we can use Kalman decomposition to bring

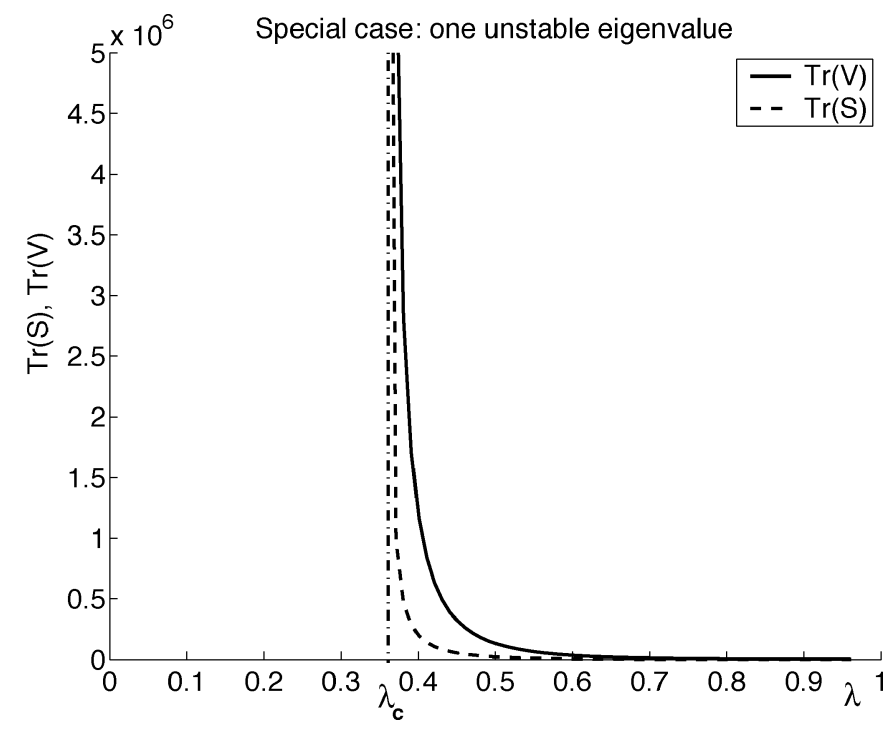

Fig. 3. Example of transition to instability with a single unstable eigenvalue in the MIMO case. The dashed line shows the asymptotic value of the trace of lower bound $(\bar{S})$, the solid line the asymptotic value of trace of the upper bound $(\bar{V})$, and the dashed-dotted line shows the asymptote $\left(\lambda_{c}\right)$.

to zero the unstable part of $F$ and thereby obtain tight bounds. Fig. 3 shows a plot for the system

$$
A=\left(\begin{array}{ccc}
1.25 & 1 & 0 \\
0 & 0.9 & 7 \\
0 & 0 & 0.6
\end{array}\right) \quad C=\left(\begin{array}{lll}
1 & 0 & 2
\end{array}\right)
$$

with $v_{t}$ and $w_{t}$ having zero mean and variance $R=2.5$ and $Q=20 \cdot I_{3 \times 3}$, respectively. This time, the asymptotic value for trace of upper and lower bound is plotted versus $\lambda$. Once again $\lambda_{c}=0.36$.

In general, $F$ cannot always be made zero and we have shown that while a lower bound on $\lambda_{c}$ can be written in closed form, an upper bound on $\lambda_{c}$ is the result of a LMI. Fig. 4 shows an example where upper and lower bounds have different convergence conditions. The system used for this simulation is

$$
A=\left(\begin{array}{cc}
1.25 & 0 \\
1 & 1.1
\end{array}\right) \quad C=\left(\begin{array}{ll}
1 & 1
\end{array}\right)
$$

with $v_{t}$ and $w_{t}$ having zero mean and variance $R=2.5$ and $Q=20 \cdot I_{2 \times 2}$, respectively.

Finally, in Fig. 5 we report results of another experiment, plotting the state estimation error for the scalar system used above at two similar values of $\lambda$, one being below and one above the critical value. We note a dramatic change in the error at $\lambda_{c} \approx 0.36$. The figure on the left shows the estimation error with $\lambda=0.3$. The figure on the right shows the estimation error for the same system evolution with $\lambda=0.4$. In the first case, the estimation error grows dramatically, making it practically useless for control purposes. In the second case, a small increase in $\lambda$ reduces the estimation error by approximately three orders of magnitude.

\section{Static Versus Dynamic Kalman Gain}

In this section, we compare the performance of filtering with static and dynamic gain for a scalar discrete system. For the static estimator, we follow the jump linear system approach of 

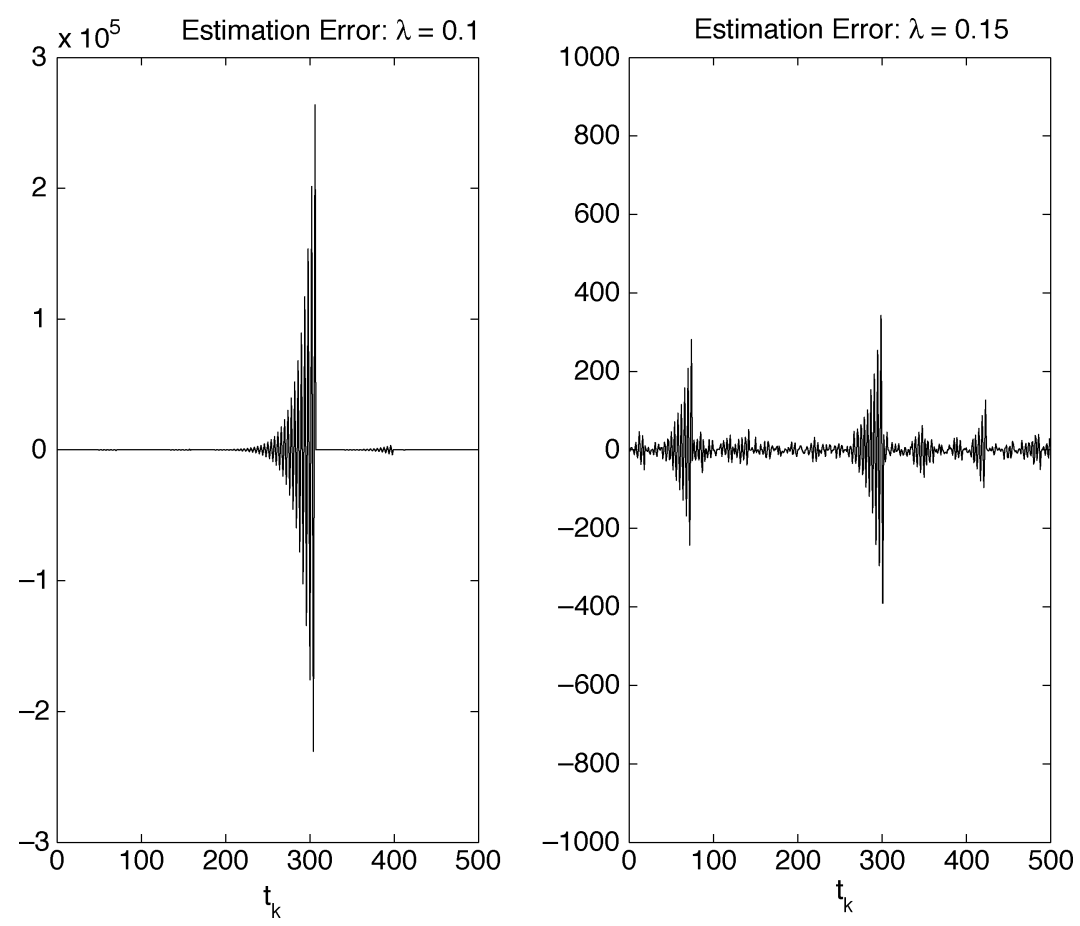

Fig. 5. Estimation error for $\lambda$ (left) below and (right) above the critical value.

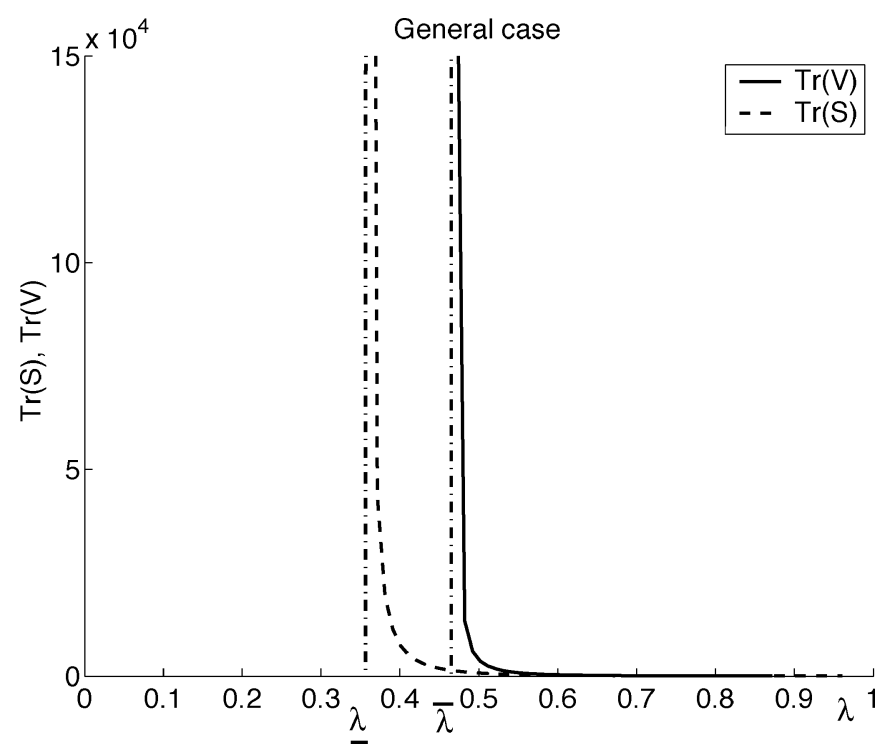

Fig. 4. Transition to instability in the general case, with arbitrary A and C. In this case, lower and upper bounds do not have the same asymptote.

[18]. The scalar static estimator case has been also worked out in [28].

Consider the dynamic state estimator

$$
\begin{aligned}
\hat{x}_{t+1}^{d} & =A \hat{x}_{t}^{d}+\gamma_{t} K_{t}^{d}\left(y_{t}-\hat{y}_{t}\right) \\
K_{t}^{d} & =A P_{t} C^{\prime}\left(C P_{t} C^{\prime}+R\right)^{-1} \\
P_{t+1} & =A P_{t} A^{\prime}+Q-\gamma_{t} K_{t}^{d} C P_{t} A^{\prime}
\end{aligned}
$$

where the Kalman gain $K_{t}^{d}$ is time-varying. Also consider the static state estimator

$$
\hat{x}_{t+1}^{s}=A \hat{x}_{t}^{d}+\gamma_{t} K_{s}\left(y_{t}-\hat{y}_{t}\right)
$$

where the estimator gain $K_{s}$ is constant. If no data arrives, i.e., $\gamma_{t}=0$, both estimators simply propagate the state estimate of the previous time-step.

The performance of the dynamic state estimator (23) has been analyzed in the previous sections. The performance of static state estimator (24), instead, can be readily obtained using jump linear system theory [16], [18]. To do so, let us consider the estimator error $e_{t+1}^{s} \triangleq x_{t+1}-\hat{x}_{t+1}^{s}$. Substituting (1) for $x_{t+1}$ and (24) for $\hat{x}_{t+1}^{s}$, we obtain the dynamics of the estimation error

$$
e_{t+1}^{s}=\left(A-\gamma_{t} K_{s} C\right) e_{t}^{s}+v_{t}+\gamma_{t} K_{s} w_{t}
$$

Using the same notation of $[18$, Ch. 6], where the author considers the general system

$$
z_{k+1}=\Phi\left(r_{k}\right) z_{k}+\Gamma\left(r_{k}\right) e_{k}
$$

system (25) can be seen as jump linear system switching between two states $r_{k} \in\{1,2\}$ given by

$$
\begin{array}{lrl}
\Phi(1)=A-K_{s} C & \Gamma(1)=\left[\begin{array}{ll}
1 & K_{s}
\end{array}\right] \\
\Phi(2)=A & \Gamma(2)=\left[\begin{array}{ll}
1 & 0
\end{array}\right]
\end{array}
$$

where the noise covariance $\mathbb{E}\left[e_{k} e_{k}^{\prime}\right]=R_{e}$, the transition probability matrix $Q_{\pi}$ and the steady-state probability distribution $\pi^{\infty}$ are given by

$$
R_{e}=\left[\begin{array}{ll}
Q & 0 \\
0 & R
\end{array}\right] Q_{\pi}=\left[\begin{array}{ll}
\lambda & 1-\lambda \\
\lambda & 1-\lambda
\end{array}\right] \pi^{\infty}=\left[\begin{array}{ll}
\lambda & 1-\lambda
\end{array}\right] .
$$




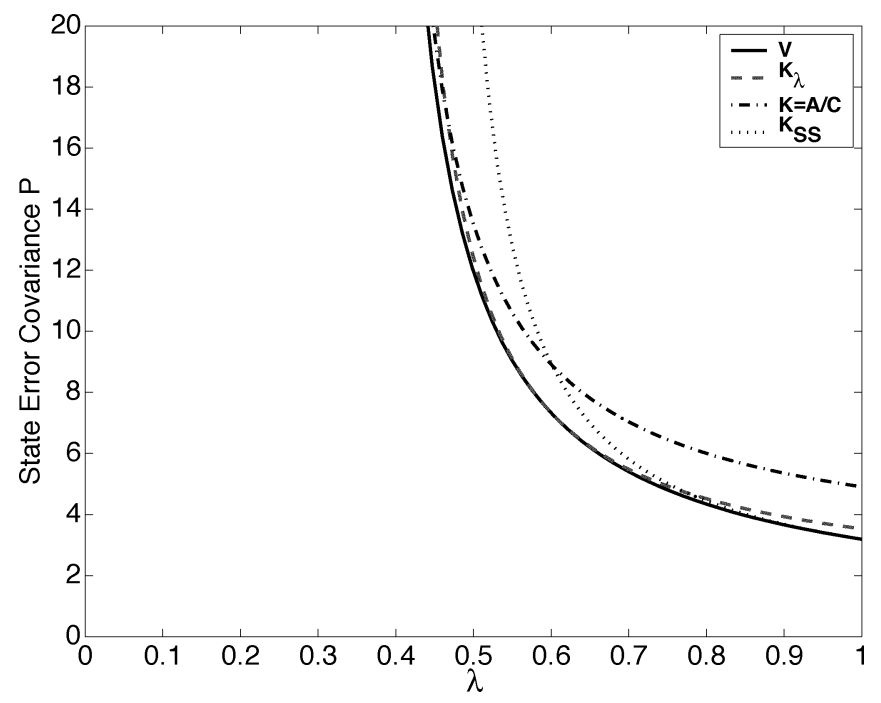

Fig. 6. Error covariance bound $\bar{V}$ for dynamic predictor obtained from our theory and steady-state error covariance for three natural static predictors obtained from JLS theory.

Following the methodology proposed in [18], it is possible to show that the previous system is mean square stable, i.e., $\lim _{t \rightarrow \infty} \mathbb{E}\left[\left(e_{t}^{s}\right)^{\prime} e_{t}^{s}\right]=0$ if and only if the transition probability is

$$
\lambda<\lambda_{s}=\frac{1}{1-\left(1-\frac{K_{s} C}{A}\right)^{2}}\left(1-\frac{1}{A^{2}}\right) .
$$

If the system is mean square stable, the steady-state error covariance $P_{\infty}^{s}=\lim _{t \rightarrow \infty} \mathbb{E}\left[e_{t}^{s}\left(e_{t}^{s}\right)^{\prime}\right]$ is given by

$$
P_{\infty}^{s}=\frac{Q+K_{s}^{2} R}{1-\lambda\left(A-K_{s} C\right)^{2}-(1-\lambda) A^{2}} .
$$

Calculations to obtain (26) and (27) are tedious but straightforward, therefore, they are omitted.

It is immediately evident that the critical transition probability $\lambda_{s}$ of the estimator (24) using a static gain is always greater than the critical transition probability $\lambda_{c}$ of the estimator (23) which adopts a dynamic gain, in fact

$$
\lambda_{s}=\lambda_{c} \frac{1}{1-\left(1-\frac{K_{s} C}{A}\right)^{2}}
$$

and the two probabilities are equal only when $K_{s}=A / C$.

A natural choice for the static estimator gain $K_{s}$ is the steadystate Kalman gain $K_{S S}$ of the closed-loop system $(r=1)$, which is always different from $A / C$. For the scalar system considered in the previous section, where $A=-1.5, C=1$, $Q=1, R=2.5$, this is given by $K_{S S}=-0.70$, while the gain for largest mean square stability range is $K_{s}=A / C=-1.25$. In the special case when the arrival probability is known, another natural choice for the estimator gain $K$ is obtained by substituting the error covariance solution of $\bar{P}=g_{\lambda}(\bar{P})$ into the equation for the Kalman filter gain $K_{\lambda}=A \bar{P} C^{\prime}\left(C \bar{P} C^{\prime}+R\right)^{-1}$. For example, assuming $\lambda=0.6$, then $\bar{P}=7.32$ and $K_{\lambda}=$ -0.93 . Fig. 6 shows all of these cases, comparing them with the upper bound on the state error covariance $\bar{V}$ of the dynamic estimator (23) that can be computed as indicated in Theorem 6.

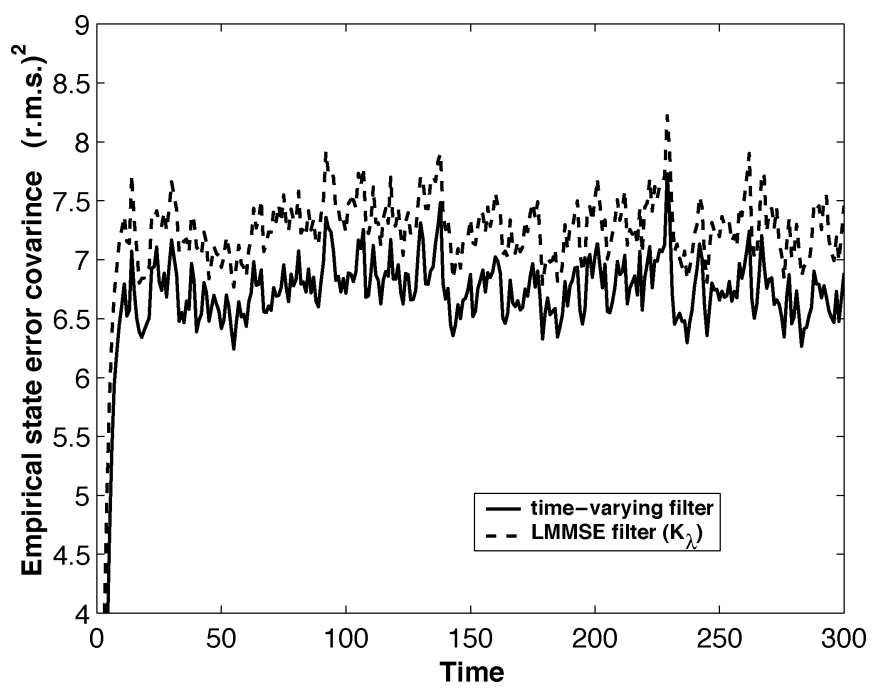

Fig. 7. Empirical state error covariance of our time-varying filter and the linear minimum mean square error estimator [17] obtained by using the optimal static kalman gain $K_{\lambda}$. The curves are obtained by averaging 10000 Monte Carlo simulations for $t=1, \ldots, 300$, with the values of the input noise $\left(v_{t}, w_{t}\right)$ and the arrival sequence $\gamma_{t}$ generated randomly. Both filters were compared under the same conditions.

The steady-state error covariance of the static predictor for the three different gains is always greater then our upper bound $\bar{V}$. This is not surprising, since the dynamic estimator is optimal over all possible estimators as shown in Section II. Note that the static predictor with static gain $K_{\lambda}$ (designed for $\lambda=0.6$ ) achieves the same state error covariance predicted by our upper bound for the optimal dynamic filter when $\lambda=0.6$. However, the empirical error state covariance is on average better than the static filter, as shown in Fig. 7. This is to be expected, since the solution of MARE gives only an upper bound of the true expected state covariance of the time-varying filter. Moreover, it is worth stressing that if the arrival probability is different from the one used to design the static gain, the performance of the static filter will degrade considerably, while the time-varying filter will still perform optimally since it does not require knowledge of $\lambda$. From this example, it seems that the upper bound for the dynamic estimator $\bar{V}$ gives an estimate of the minimum steady-state covariance that can be achieved with a static estimator for any given arrival probability if the static gain $K_{s}$ is chosen optimally. Then, the MARE could be used to find the minimum steady-state covariance and then the corresponding steady-state modified Kalman gain, thus providing an useful tool for optimal static estimator design. Future work will explore this possibility.

\section{CONCLUSION}

In this paper, we have presented an analysis of Kalman filtering in the setting of intermittent observations. We have shown how the expected estimation error covariance depends on the tradeoff between loss probability and the system dynamics. Such a result is useful to the system designer who must assess the relationship between the dynamics of the system whose state is to be estimated and the reliability of the communication channel through which that system is measured. 
Our motivating application is a distributed sensor network that collects observations and sends them to one or more central units that are responsible for estimation and control. For example, in a pursuit evasion game in which mobile pursuers perform their control actions based on the current estimate of the positions of both pursuers and evaders, the sensing capability of each pursuer is generally limited, and an embedded sensor network is essential for providing a larger overall view of the terrain. The results that we have presented here can aid the designer of the sensor network in the choice of the number and disposition of the sensors.

This application also suggests a number of interesting directions for further work. For example, although we have assumed independent Bernoulli probabilities for the observation events, in the sensor network there will generally be temporal and spatial sources of variability that lead to correlations among these events. While it is possible to compute posterior state estimates in such a setting, it would be of interest to see if a priori bounds of the kind that we have obtained here can be obtained in this case. Similarly, in many situations there may be correlations between the states and the observation events; for example, such correlations will arise in the pursuit evasion game when the evaders move near the boundaries of the sensor network. Finally, the sensor network setting also suggests the use of smoothing algorithms in addition to the filtering algorithms that have been our focus here. In particular, we may be willing to tolerate a small amount of additional delay to wait for the arrival of a sensor measurement, if that measurement is expected to provide a significant reduction in uncertainty. Thus, we would expect that the tradeoff that we have studied here between loss probability and the system dynamics should also be modulated in interesting ways by the delay due to smoothing.

We also remark that the assumption of modeling the arrival of observations as a Bernoulli i.i.d. process can be clearly improved upon. For example, one can imagine situations where some of the sensing is done locally and therefore measurements are available at all sampling times, while measurements taken at distant locations are available at irregular intervals. This would translate in different dropping rates for different channels. We have focused on providing a basic result upon which more sophisticated models can be built and analyzed.

\section{APPENDIX A}

In order to give complete proofs of our main theorems, we need to prove some preliminary lemmas. The first one shows some useful properties of the MARE.

Lemma 1: Let the operator

$$
\phi(K, X)=(1-\lambda)\left(A X A^{\prime}+Q\right)+\lambda\left(F X F^{\prime}+V\right)
$$

where $F=A+K C, V=Q+K R K^{\prime}$. Assume $X \in \mathbb{S}=\{S \in$ $\left.\mathbb{R}^{n \times n} \mid S \geq 0\right\}, R>0, Q \geq 0$, and $\left(A, Q^{1 / 2}\right)$ is controllable. Then, the following facts are true.
a) With $K_{X}=-A X C^{\prime}\left(C X C^{\prime}+R\right)^{-1}, g_{\lambda}(X)=$ $\phi\left(K_{X}, X\right)$.
b) $g_{\lambda}(X)=\min _{K} \phi(K, X) \leq \phi(K, X), \forall K$.
c) If $X \leq Y$, then $g_{\lambda}(X) \leq \bar{g}_{\lambda}(Y)$.
d) If $\lambda_{1} \leq \lambda_{2}$ then $g_{\lambda_{1}}(X) \geq g_{\lambda_{2}}(X)$.

e) If $\alpha \in[0,1]$, then $g_{\lambda}(\alpha X+(1-\alpha) Y) \geq \alpha g_{\lambda}(X)+(1-$ $\alpha) g_{\lambda}(Y)$.

f) $g_{\lambda}(X) \geq(1-\lambda) A X A^{\prime}+Q$.

g) If $\bar{X} \geq g_{\lambda}(\bar{X})$, then $\bar{X}>0$.

h) If $X$ is a random variable, then $(1-\lambda) A \mathbb{E}[X] A^{\prime}+Q \leq$ $\mathbb{E}\left[g_{\lambda}(X)\right] \leq g_{\lambda}(\mathbb{E}[X])$.

Proof:

a) Define $F_{X}=A+K_{X} C$, and observe that

$$
\begin{aligned}
F_{X} X C^{\prime}+K_{X} R & =\left(A+K_{X} C\right) X C^{\prime}+K_{X} R \\
& =A X C^{\prime}+K_{X}\left(C X C^{\prime}+R\right)=0 .
\end{aligned}
$$

Next, we have

$$
\begin{aligned}
g_{\lambda}(X)= & (1-\lambda)\left(A X A^{\prime}+Q\right) \\
& +\lambda\left(A X A^{\prime}+Q-A X C^{\prime}\left(C X C^{\prime}+R\right)^{-1} C X A^{\prime}\right) \\
= & (1-\lambda)\left(A X A^{\prime}+Q\right)+\lambda\left(A X A^{\prime}+Q+K_{X} C X A^{\prime}\right) \\
= & (1-\lambda)\left(A X A^{\prime}+Q\right)+\lambda\left(F_{X} X A^{\prime}+Q\right) \\
= & (1-\lambda)\left(A X A^{\prime}+Q\right) \\
& +\lambda\left(F_{X} X A^{\prime}+Q\right)+\left(F_{X} X C^{\prime}+K_{X} R\right) K^{\prime} \\
= & \phi\left(K_{X}, X\right) .
\end{aligned}
$$

b) Let $\psi(K, X)=(A+K C) X(A+K C)^{\prime}+K R K^{\prime}+Q$. Note that

$$
\begin{aligned}
\operatorname{argmin}_{K} \phi(K, X) & =\operatorname{argmin}_{K} F X F^{\prime}+V \\
& =\operatorname{argmin}_{K} \psi(X, K) .
\end{aligned}
$$

Since $X, R \geq 0, \phi(K, X)$ is quadratic and convex in the variable $\bar{K}$, therefore, the minimizer can be found by solving $(\partial \psi(K, X)) /(\partial K)=0$, which gives

$2(A+K C) X C^{\prime}+2 K R=0 \Longrightarrow K=-A X C^{\prime}\left(C X C^{\prime}+R\right)^{-1}$.

Since the minimizer corresponds to $K_{X}$ defined above, the fact follows from fact (1).

c) Note that $\phi(K, X)$ is affine in $X$. Suppose $X \leq Y$. Then

$g_{\lambda}(X)=\phi\left(K_{X}, X\right) \leq \phi\left(K_{Y}, X\right) \leq \phi\left(K_{Y}, Y\right)=g_{\lambda}(Y)$.

This completes the proof.

d) Note that $A X C^{\prime}\left(C X C^{\prime}+R\right)^{-1} C X A \geq 0$. Then

$$
\begin{aligned}
g_{\lambda_{1}}(X) & =A X A^{\prime}+Q-\lambda_{1} A X C^{\prime}\left(C X C^{\prime}+R\right)^{-1} C X A \\
& \geq A X A^{\prime}+Q-\lambda_{2} A X C^{\prime}\left(C X C^{\prime}+R\right)^{-1} C X A \\
& =g_{\lambda_{2}}(X) .
\end{aligned}
$$

e) Let $Z=\alpha X+(1-\alpha) Y$ where $\alpha \in[0,1]$. Then, we have

$$
\begin{aligned}
g_{\lambda}(Z)= & \phi\left(K_{Z}, Z\right) \\
= & \alpha\left(A+K_{Z} C\right) X\left(A+K_{Z} C\right)^{\prime} \\
& +(1-\alpha)\left(A+K_{Z} C\right) Y\left(A+K_{Z} C\right)^{\prime} \\
& +(\alpha+1-\alpha)\left(K_{Z} R K_{Z}^{\prime}+Q\right) \\
= & \alpha \phi\left(K_{Z}, X\right)+(1-\alpha) \phi\left(K_{Z}, Y\right) \\
\geq & \alpha \phi\left(K_{X}, X\right)+(1-\alpha) \phi\left(K_{Y}, Y\right) \\
= & \alpha g_{\lambda}(X)+(1-\alpha) g_{\lambda}(Y) .
\end{aligned}
$$


f) Note that $F_{X} X F_{X}^{\prime} \geq 0$ and $K R K^{\prime} \geq 0$, for all $K$ and $X$. Then

$$
\begin{aligned}
g_{\lambda_{1}}(X)= & \phi\left(K_{X}, X\right) \\
= & (1-\lambda)\left(A X A^{\prime}+Q\right) \\
& +\lambda\left(F_{X} X F_{X}^{\prime}+K_{X} R K_{X}^{\prime}+Q\right) \\
\geq & (1-\lambda)\left(A X A^{\prime}+Q\right)+\lambda Q \\
= & (1-\lambda) A X A^{\prime}+Q .
\end{aligned}
$$

g) From fact f), it follows that $\bar{X} \geq g_{\lambda_{1}}(\bar{X}) \geq(1-$ $\lambda) A \bar{X} A^{\prime}+Q$. Let $\hat{X}$ such that $\hat{X}=(1-\lambda) A \hat{X} A^{\prime}+Q$. Such $\hat{X}$ must clearly exist. Therefore, $\bar{X}-\hat{X} \geq$ $(1-\lambda) A(\bar{X}-\hat{X}) A^{\prime} \geq 0$. Moreover, the matrix $\overline{\hat{X}}$ solves the Lyapunov Equation $\hat{X}=\tilde{A} \hat{X} \tilde{A}^{\prime}+Q$ where $\tilde{A}=\sqrt{1-\lambda} A$. Since $\left(\tilde{A}, Q^{(1 / 2)}\right)$ is detectable, it follows that $\hat{X}>0$ and so $\bar{X}>0$, which proves the fact.

h) Using fact f) and linearity of expectation, we have

$$
\mathbb{E}\left[g_{\lambda}(X)\right] \geq \mathbb{E}\left[(1-\lambda) A X A^{\prime}+Q\right]=(1-\lambda) A \mathbb{E}[X] A^{\prime}+Q
$$

Fact e) implies that the operator $g_{\lambda}()$ is concave, therefore by Jensen's Inequality, we have $\mathbb{E}\left[g_{\lambda}(X)\right] \leq g_{\lambda}(\mathbb{E}[X])$

Lemma 2: Let $X_{t+1}=h\left(X_{t}\right)$ and $Y_{t+1}=h\left(Y_{t}\right)$. If $h(X)$ is a monotonically increasing function, then

$$
\begin{aligned}
X_{1} \geq X_{0} \Rightarrow X_{t+1} \geq X_{t} & & \forall t \geq 0 \\
X_{1} \leq X_{0} \Rightarrow X_{t+1} \leq X_{t} & & \forall t \geq 0 \\
X_{0} \leq Y_{0} \Rightarrow X_{t} \leq Y_{t} & & \forall t \geq 0 .
\end{aligned}
$$

Proof: This lemma can be readily proved by induction. It is true for $t=0$, since $X_{1} \geq X_{0}$ by definition. Now, assume that $X_{t+1} \geq X_{t}$, then $X_{t+2}=h\left(X_{t+1}\right) \geq h\left(X_{t}\right)=X_{t+1}$ because of monotonicity of $h(\cdot)$. The proof for the other two cases is analogous.

It is important to note that while in the scalar case $X \in \mathbb{R}$ either $h(X) \leq X$ or $h(X) \geq X$; in the matrix case $X \in \mathbb{R}^{n \times n}$, it is not generally true that either $h(X) \geq X$ or $h(X) \leq X$. This is the source of the major technical difficulty for the proof of convergence of sequences in higher dimensions. In this case, convergence of a sequence $\left\{X_{t}\right\}_{0}^{\infty}$ is obtained by finding two other sequences, $\left\{Y_{t}\right\}_{0}^{\infty},\left\{Z_{t}\right\}_{0}^{\infty}$ that bound $X_{t}$, i.e., $Y_{t} \leq X_{t} \leq$ $Z_{t}, \forall t$, and then by showing that these two sequences converge to the same point.

The next two Lemmas show that when the MARE has a solution $\bar{P}$, this solution is also stable, i.e., every sequence based on the difference Riccati equation $P_{t+1}=g_{\lambda}\left(P_{t}\right)$ converges to $\bar{P}$ for all initial positive semidefinite conditions $P_{0} \geq 0$.

Lemma 3: Define the linear operator

$$
\mathcal{L}(Y)=(1-\lambda)\left(A Y A^{\prime}\right)+\lambda\left(F Y F^{\prime}\right)
$$

Suppose there exists $\bar{Y}>0$ such that $\bar{Y}>\mathcal{L}(\bar{Y})$. a) For all $W \geq 0$

$$
\lim _{k \rightarrow \infty} \mathcal{L}^{k}(W)=0 .
$$

b) Let $U \geq 0$ and consider the linear system

$$
Y_{k+1}=\mathcal{L}\left(Y_{k}\right)+U \quad \text { initialized at } Y_{0} .
$$

Then, the sequence $Y_{k}$ is bounded.

Proof:

a) First observe that $0 \leq \mathcal{L}(Y)$ for all $0 \leq Y$. Also, $X \leq Y$ implies $\mathcal{L}(X) \leq \overline{\mathcal{L}}(Y)$. Choose $0 \leq r<1$ such that $\mathcal{L}(\bar{Y})<r \bar{Y}$. Choose $0 \leq m$ such that $W \leq m \bar{Y}$. Then

$$
0 \leq \mathcal{L}^{k}(W) \leq m \mathcal{L}^{k}(\bar{Y})<m r^{k} \bar{Y}
$$

The assertion follows when we take the limit $r \rightarrow \infty$, on noticing that $0 \leq r<1$.

b) The solution of the linear iteration is

$$
\begin{aligned}
Y_{k} & =\mathcal{L}^{k}\left(Y_{0}\right)+\sum_{t=0}^{k-1} \mathcal{L}^{t}(U) \\
& \leq\left(m_{Y_{0}} r^{k}+\sum_{t=0}^{k-1} m_{U} r^{t}\right) \bar{Y} \\
& \leq\left(m_{Y_{0}} r^{k}+\frac{m_{U}}{1-r}\right) \bar{Y} \\
& \leq\left(m_{Y_{0}}+\frac{m_{U}}{1-r}\right) \bar{Y}
\end{aligned}
$$

proving the claim.

Lemma 4: Consider the operator $\phi(K, X)$ defined in (28). Suppose there exists a matrix $\bar{K}$ and a positive-definite matrix $\bar{P}$ such that

$$
\bar{P}>0 \quad \text { and } \quad \bar{P}>\phi(\bar{K}, \bar{P}) .
$$

Then, for any $P_{0}$, the sequence $P_{t}=g_{\lambda}^{t}\left(P_{0}\right)$ is bounded, i.e., there exists $M_{P_{0}} \geq 0$ dependent of $P_{0}$ such that

$$
P_{t} \leq M \quad \forall t
$$

Proof: First, define the matrices $\bar{F}=A+\bar{K} C$ and consider the linear operator

$$
\mathcal{L}(Y)=(1-\lambda)\left(A Y A^{\prime}\right)+\lambda\left(\bar{F} Y \bar{F}^{\prime}\right)
$$

Observe that

$$
\bar{P}>\phi(\bar{K}, \bar{P})=\mathcal{L}(\bar{P})+Q+\lambda \bar{K} R \bar{K}^{\prime} \geq \mathcal{L}(\bar{P}) .
$$

Thus, $\mathcal{L}$ meets the condition of Lemma 3. Finally, using fact b) in Lemma 1 we have

$P_{t+1}=g_{\lambda}\left(P_{t}\right) \leq \phi\left(\bar{K}, P_{t}\right)=\mathcal{L} P_{t}+Q+\lambda \bar{K} R \bar{K}^{\prime}=\mathcal{L}\left(P_{t}\right)+U$. 
Since $U=\lambda \bar{K} R \bar{K}^{\prime}+Q \geq 0$, using Lemma 3, we conclude that the sequence $P_{t}$ is bounded.

We are now ready to give proofs for Theorems 1-4.

\section{A. Proof of Theorem 1}

a) We first show that the modified Riccati difference equation initialized at $Q_{0}=0$ converges. Let $Q_{k}=g_{\lambda}^{k}(0)$. Note that $0=Q_{0} \leq Q_{1}$. It follows from Lemma 1c) that

$$
Q_{1}=g_{\lambda}\left(Q_{0}\right) \leq g_{\lambda}\left(Q_{1}\right)=Q_{2} .
$$

A simple inductive argument establishes that

$$
0=Q_{0} \leq Q_{1} \leq Q_{2} \leq \cdots \leq M_{Q_{0}} .
$$

Here, we used Lemma 4 to bound the trajectory. We now have a monotone nondecreasing sequence of matrices bounded above. It is a simple matter to show that the sequence converges, i.e.,

$$
\lim _{k \rightarrow \infty} Q_{k}=\bar{P} \text {. }
$$

Also, we see that $\bar{P}$ is a fixed point of the modified Riccati iteration

$$
\bar{P}=g_{\lambda}(\bar{P})
$$

which establishes that it is $a$ positive-semidefinite solution of the MARE.

Next, we show that the Riccati iteration initialized at $R_{0} \geq \bar{P}$ also converges, and to the same limit $\bar{P}$. First, define the matrices

$$
\bar{K}=-A \bar{P} C^{\prime}\left(C \bar{P} C^{\prime}+R\right)^{-1} \quad \bar{F}=A+\bar{K} C
$$

and consider the linear operator

$$
\hat{\mathcal{L}}(Y)=(1-\lambda)\left(A Y A^{\prime}\right)+\lambda\left(\bar{F} Y \bar{F}^{\prime}\right) .
$$

Observe that

$$
\bar{P}=g_{\lambda}(\bar{P})=\mathcal{L}(\bar{P})+Q+\bar{K} R \bar{K}^{\prime}>\hat{\mathcal{L}}(\bar{P}) .
$$

Thus, $\hat{\mathcal{L}}$ meets the condition of Lemma 3. Consequently, for all $Y \geq 0$

$$
\lim _{k \rightarrow \infty} \hat{\mathcal{L}}^{k}(Y)=0 .
$$

Now, suppose $R_{0} \geq \bar{P}$. Then

$$
R_{1}=g_{\lambda}\left(R_{0}\right) \geq g_{\lambda}(\bar{P})=\bar{P} .
$$

A simple inductive argument establishes that

$$
R_{k} \geq \bar{P} \quad \forall k .
$$

Observe that

$$
\begin{aligned}
0 \leq\left(R_{k+1}-\bar{P}\right)= & g_{\lambda}\left(R_{k}\right)-g_{\lambda}(\bar{P}) \\
= & \phi\left(K_{R_{k}}, R_{k}\right)-\phi\left(K_{\bar{P}}, \bar{P}\right) \\
\leq & \phi\left(K_{\bar{P}}, R_{k}\right)-\phi\left(K_{\bar{P}}, \bar{P}\right) \\
= & (1-\lambda) A\left(R_{k}-\bar{P}\right) A^{\prime} \\
& +\lambda F_{\bar{P}}\left(R_{k}-\bar{P}\right) F_{\bar{P}}^{\prime} \\
= & \hat{\mathcal{L}}\left(R_{k}-\bar{P}\right) .
\end{aligned}
$$

Then, $0 \leq \lim _{k \rightarrow \infty}\left(R_{k+1}-\bar{P}\right) \leq 0$, proving the claim.
We now establish that the Riccati iteration converges to $\bar{P}$ for all initial conditions $P_{0} \geq 0$. Define $Q_{0}=0$ and $R_{0}=P_{0}+\bar{P}$. Consider three Riccati iterations, initialized at $Q_{0}, P_{0}$, and $R_{0}$. Note that

$$
Q_{0} \leq P_{0} \leq R_{0}
$$

It then follows from Lemma 2 that

$$
Q_{k} \leq P_{k} \leq R_{k} \quad \forall k .
$$

We have already established that the Riccati equations $P_{k}$ and $R_{k}$ converge to $\bar{P}$. As a result, we have

$$
\bar{P}=\lim _{k \rightarrow \infty} P_{k} \leq \lim _{k \rightarrow \infty} Q_{k} \leq \lim _{k \rightarrow \infty} R_{k}=\bar{P}
$$

proving the claim.

b) Finally, we establish that the MARE has a unique positive-semidefinite solution. To this end, consider $\hat{P}=g_{\lambda}(\hat{P})$ and the Riccati iteration initialized at $P_{0}=\hat{P}$. This yields the constant sequence

$$
\hat{P}, \hat{P}, \ldots
$$

However, we have shown that every Riccati iteration converges to $\bar{P}$. Thus, $\bar{P}=\hat{P}$.

\section{B. Proof of Theorem 2}

First, we note that the two cases expressed by the theorem are indeed possible. If $\lambda=1$, the modified Riccati difference equation reduces to the standard Riccati difference equation, which is known to converge to a fixed point, under the theorem's hypotheses. Hence, the covariance matrix is always bounded in this case, for any initial condition $P_{0} \geq 0$. If $\lambda=0$, then we reduce to open-loop prediction, and if the matrix $A$ is unstable, then the covariance matrix diverges for some initial condition $P_{0} \geq 0$. Next, we show the existence of a single point of transition between the two cases. Fix a $0<\lambda_{1} \leq 1$ such that $\mathbb{E}_{\lambda_{1}}\left[P_{t}\right]$ is bounded for any initial condition $P_{0} \geq 0$. Then, for any $\lambda_{2} \geq \lambda_{1} \mathbb{E}_{\lambda_{2}}\left[P_{t}\right]$ is also bounded for all $P_{0} \geq 0$. In fact, we have

$$
\begin{aligned}
\mathbb{E}_{\lambda_{1}}\left[P_{t+1}\right]= & \mathbb{E}_{\lambda_{1}}\left[A P_{t} A^{\prime}+Q+\right. \\
& \left.\quad-\gamma_{t+1} A P_{t} C^{\prime}\left(C P_{t} C^{\prime}+R\right)^{-1} C P_{t} A\right] \\
= & \mathbb{E}\left[A P_{t} A^{\prime}+Q+\right. \\
& \left.\quad-\lambda_{1} A P_{t} C^{\prime}\left(C P_{t} C^{\prime}+R\right)^{-1} C P_{t} A\right] \\
= & \mathbb{E}\left[g_{\lambda_{1}}\left(P_{t}\right)\right] \\
\geq & \mathbb{E}\left[g_{\lambda_{2}}\left(P_{t}\right)\right] \\
= & \mathbb{E}_{\lambda_{2}}\left[P_{t+1}\right]
\end{aligned}
$$

where we exploited fact d) of Lemma 1 to write the previous inequality. We can now choose

$$
\lambda_{c}=\left\{\inf \lambda^{*}: \lambda>\lambda^{*} \Rightarrow \mathbb{E}_{\lambda}\left[P_{t}\right] \text { is bounded, for all } P_{0} \geq 0\right\}
$$

completing the proof.

\section{Proof of Theorem 3}

Define the Lyapunov operator $m(X)=\tilde{A} X \tilde{A}^{\prime}+Q$ where $\tilde{A}=\sqrt{1-\lambda} A$. If $\left(A, Q^{1 / 2}\right)$ is controllable, also $\left(\tilde{A}, Q^{1 / 2}\right)$ is 
controllable. Therefore, it is well known that $\hat{S}=m(\hat{S})$ has a unique strictly positive definite solution $\hat{S}>0$ if and only if $\max _{i}\left|\sigma_{i}(\tilde{A})\right|<1$, i.e. $\sqrt{1-\lambda} \max _{i}\left|\sigma_{i}(A)\right|<1$, from which follows $\underline{\lambda}=1-\left(1 / \alpha^{2}\right)$. If $\max _{i}\left|\sigma_{i}(\tilde{A})\right| \geq 1$ it is also a well-known fact that there is no positive-semidefinite fixed point to the Lyapunov equation $\hat{S}=m(\hat{S})$, since $\left(\tilde{A}, Q^{1 / 2}\right)$ is controllable.

Let us consider the difference equation $S_{t+1}=m\left(S_{t}\right), S_{0}=$ 0 . It is clear that $S_{0}=0 \leq Q=S_{1}$. Since the operator $m()$ is monotonic increasing, by Lemma 2 it follows that the sequence $\left\{S_{t}\right\}_{0}^{\infty}$ is monotonically increasing, i.e., $S_{t+1} \geq S_{t}$ for all $t$. If $\lambda<\lambda$ this sequence does not converge to a finite matrix $\bar{S}$, otherwise by continuity of the operator $m$ we would have $\bar{S}=m(\bar{S})$, which is not possible. Since it is easy to show that a monotonically increasing sequence $S_{t}$ that does not converge is also unbounded, then we have

$$
\lim _{t \rightarrow \infty} S_{t}=\infty .
$$

Let us consider now the mean covariance matrix $\mathbb{E}\left[P_{t}\right]$ initialized at $\mathbb{E}\left[P_{0}\right] \geq 0$. Clearly, $0=S_{0} \leq \mathbb{E}\left[P_{0}\right]$. Moreover, it is also true that $S_{t} \leq \mathbb{E}\left[P_{t}\right]$ implies

$$
\begin{aligned}
S_{t+1} & =(1-\lambda) A S_{t} A^{\prime}+Q \\
& \leq(1-\lambda) A \mathbb{E}\left[P_{t}\right] A^{\prime}+Q \\
& \leq \mathbb{E}\left[g_{\lambda}\left(P_{t}\right)\right] \\
& =\mathbb{E}\left[P_{t+1}\right]
\end{aligned}
$$

where we used fact $h$ ) from Lemma 1. By induction, it is easy to show that

$$
S_{t} \leq \mathbb{E}\left[P_{t}\right] \forall t, \forall \mathbb{E}\left[P_{0}\right] \geq 0 \Longrightarrow \lim _{t \rightarrow \infty} \mathbb{E}\left[P_{t}\right] \geq \lim _{t \rightarrow \infty} S_{t}=\infty .
$$

This implies that for any initial condition $\mathbb{E}\left[P_{t}\right]$ is unbounded for any $\lambda<\underline{\lambda}$, therefore, $\underline{\lambda} \leq \lambda_{c}$, which proves the first part of the Theorem.

Now, consider the sequence $V_{t+1}=g_{\lambda}\left(V_{t}\right), V_{0}=\mathbb{E}\left[P_{0}\right] \geq$ 0 . Clearly, $\mathbb{E}\left[P_{t}\right] \leq V_{t}$ implies

$$
\begin{aligned}
\mathbb{E}\left[P_{t+1}\right] & =\mathbb{E}\left[g_{\lambda}\left(P_{t}\right)\right] \\
& \leq g_{\lambda}\left(\mathbb{E}\left[P_{t}\right]\right) \\
& \leq\left[g_{\lambda}\left(V_{t}\right)\right] \\
& =V_{t+1}
\end{aligned}
$$

where we used facts c) and h) from Lemma 1 . Then a simple induction argument shows that $V_{t} \geq \mathbb{E}\left[P_{t}\right]$ for all $t$. Let us consider the case $\lambda>\bar{\lambda}$, therefore, there exists $\hat{X}$ such that $\hat{X} \geq g_{\lambda}(\hat{X})$. By Lemma $1(\mathrm{~g}) \bar{X}>0$, therefore, all hypotheses of Lemma 3 are satisfied, which implies that

$$
\mathbb{E}\left[P_{t}\right] \leq V_{t} \leq M_{V_{0}} \quad \forall t .
$$

This shows that $\lambda_{c} \leq \bar{\lambda}$ and concludes the proof of the theorem.

\section{Proof of Theorem 4}

Let us consider the sequences $S_{t+1}=(1-\lambda) A S_{t} A^{\prime}+Q$, $S_{0}=0$ and $V_{t+1}=g_{\lambda}\left(V_{t}\right), V_{0}=\mathbb{E}\left[P_{0}\right] \geq 0$. Using the same induction arguments in Theorem 3 it is easy to show that

$$
S_{t} \leq \mathbb{E}\left[P_{t}\right] \leq V_{t} \quad \forall t
$$

From Theorem 1, it also follows that $\lim _{t \rightarrow \infty} V_{t}=\bar{V}$, where $\bar{V}=g_{\lambda}(V)$. As shown previously, the sequence $S_{t}$ is monotonically increasing. Also, it is bounded since $S_{t} \leq V_{t} \leq M$. Therefore, $\lim _{t \rightarrow \infty} S_{t}=\bar{S}$, and by continuity $\bar{S}=(1-\bar{\lambda}) A \bar{S} A^{\prime}+Q$ which is a Lyapunov equation. Since $\sqrt{1-\lambda} A$ is stable and $\left(A, Q^{1 / 2}\right)$ is controllable, then the solution of the Lyapunov equation is strictly positive definite, i.e., $\bar{S}>0$. Adding all of the results together, we get

$$
0<\bar{S}=\lim _{t \rightarrow \infty} S_{t} \leq \lim _{t \rightarrow \infty} \mathbb{E}\left[P_{t}\right] \leq \lim _{t \rightarrow \infty} V_{t}=\bar{V}
$$

which concludes the proof.

\section{ACKNOWLEDGMENT}

The authors would like to thank the anonymous reviewer for the comments that helped to improve the quality of the manuscript.

\section{REFERENCES}

[1] Smart Dust Project Home Page. Univ. California, Berkeley. [Online]http://robotics.eecs.berkeley.edu/ pister/SmartDust/

[2] NEST Project at Berkeley Home Page. Univ. California, Berkeley. [Online]http://webs.cs.berkeley.edu/nest-index.html

[3] Seismic Sensor Research at Berkeley Home Page. Univ. California, Berkeley. [Online]http://www.berkeley.edu/news/media/releases/2001/12/13\_snsor.html

[4] P. Varaiya, "Smart cars on smart roads: Problems of control," IEEE Trans. Automat. Contr., vol. 38, pp. 195-207, Feb. 1993.

[5] J. Lygeros, D. N. Godbole, and S. S. Sastry, "Verified hybrid controllers for automated vehicles," IEEE Trans. Automat. Contr., vol. 43, pp. 522-539, Apr. 1998.

[6] B. Sinopoli, C. Sharp, S. Schaffert, L. Schenato, and S. Sastry, "Distributed control applications within sensor networks," Proc. IEEE, vol. 91, pp. 1235-1246, Aug. 2003.

[7] R. E. Kalman, "A new approach to linear filtering and prediction problems," Trans. ASME_J. Basic Eng. Automat. Control, vol. 82, no. D, pp. 35-45, Mar. 1960.

[8] P. S. Maybeck, Stochastic Models, Estimation, and Control, ser. Mathematics in Science and Engineering. New York: Academic, 1979, vol. 141.

[9] N. Gordon, D. Salmond, and A. Smith, "A novel approach to nonlinear nongaussian bayesian state estimation," in Proc. IEEE Conf. Radar and Signal Processing, vol. 140, 1993, pp. 107-113.

[10] M. Micheli and M. I. Jordan, "Random sampling of a continiuous-time stochastic dynamical system," presented at the 15th Int. Symp. Mathematical Theory of Networks and Systems (MTNS), South Bend, IN, Aug. 2002.

[11] M. Micheli, "Random sampling of a continuous-time stochastic dynamical system: Analysis, state estimation, applications," M.S. thesis, Dept Elect. Eng., Univ. California, Berkeley, 2001.

[12] M. Athans, R. Ku, and S. B. Gershwin, "The uncertainty threshold principle, some fundamental limitations of optimal decision making under dynamic uncertainty," IEEE Trans. Automat. Contr, vol. AC-22, pp. 491-495, June 1977.

[13] R. Ku and M. Athans, "Further results on the uncertainty threshold principle," IEEE Trans. Automat. Contr., vol. AC-22, pp. 491-495, Oct. 1977.

[14] N. Nahi, "Optimal recursive estimation with uncertain observation," IEEE Trans. Inform. Theory, vol. IT-15, pp. 457-462, Apr. 1969.

[15] M. Hadidi and S. Schwartz, "Linear recursive state estimators under uncertain observations," IEEE Trans. Inform. Theory, vol. IT-24, pp 944-948, June 1979.

[16] M. Mariton, Jump Linear Systems in Automatic Control. New York: Marcel Dekker, 1990.

[17] O. Costa, "Stationary filter for linear minimum mean square error estimator of discrete-time markovian jump systems," IEEE Trans. Automat. Contr., vol. 48, pp. 1351-1356, 2002.

[18] J. Nilsson, "Real-time control systems with delays," Ph.D. dissertation, Dept. Automatic Control, Lund Inst. Technol., Lund, Sweden, 1998. 
[19] J. Nilsson, B. Bernhardsson, and B. Wittenmark, "Stochastic analysis and control of real-time systems with random time delays," Automatica, vol. 34, no. 1, pp. 57-64, Jan. 1998.

[20] Q. Ling and M. Lemmon, "Soft real-time scheduling of networked control systems with dropouts governed by a Markov chain," in Amer. Control Conf., vol. 6, Denver, CO, June 2003, pp. 4845-4550.

[21] Y. Wang, D. Ho, and X. Liu, "Variance-constrained filtering for uncertain stochastic systems with missing measurements," IEEE. Trans. Automat. Contr., vol. 48, pp. 1254-1258, July 2003.

[22] S. Smith and P. Seiler, "Estimation with lossy measurements: jump estimators for jump systems," IEEE. Trans. Automat. Contr., vol. 48, pp. 2163-2171, Dec. 2003.

[23] T. Fortmann, Y. Bar-Shalom, M. Scheffe, and S. Gelfand, "Detection thresholds for tracking in clutter-a connection between estimation and signal processing," IEEE Trans. Automat. Contr., vol. AC-30, pp. 221-228, Mar. 1985.

[24] A. Matveev and A. Savkin, "The problem of state estimation via asynchronous communication channels with irregular transmission times," IEEE Trans. Automat. Contr., vol. 48, pp. 670-676, 2003.

[25] R. H. Shumway and D. S. Stoffer, Time Series Analysis and Its Applications. New York: Springer-Verlag, 2000.

[26] S. Lauritzen, Graphical Models. New York: Clarendon, 1996.

[27] S. Boyd, L. E. Ghaoui, E. Feron, and V. Balakrishnan, Linear Matrix Inequalities in System and Control Theory. Philadelphia, PA: SIAM, June 1997.

[28] C. N. Hadjicostis and R. Touri, "Feedback control utilizing packet dropping network links," in Proc. 41st IEEE Conf. Decision and Control, vol. 2, Las Vegas, NV, Dec. 2002, pp. 1205-1210.

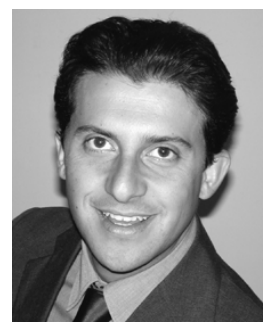

Bruno Sinopoli ( $\mathrm{S}^{\prime} 02$ ) received the Laurea degree in electrical engineering from the University of Padova, Padova, Italy, in 1998. He is currently working toward the Ph.D. degree in electrical engineering from the University of California, Berkeley.

His research interests include sensor networks, design of embedded systems from components, distributed control, and hybrid systems.

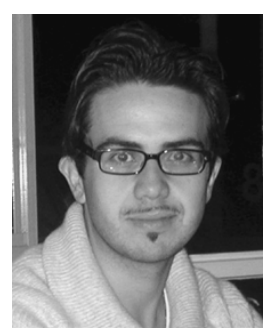

Luca Schenato (S'02-M'04) was born in Treviso, Italy, in 1974. He received the Dr.Eng. degree in electrical engineering from the University of Padova, Padova, Italy, in 1999, and the Ph.D. degree from the University of California, Berkeley, in 2003.

$\mathrm{He}$ is currently a Postdoctoral Research Assistant with the Department of Electrical Engineering, the University of California, Berkeley. His interests include modeling of biological networks, sensor networks, insect locomotion, millirobotics, and cooperative and swarm robotics.

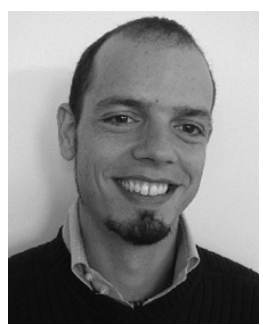

Massimo Franceschetti (M'02) received the Laurea degree (magna cum laude) in computer engineering from the University of Naples, Naples, Italy, in 1997, and the M.Sc. and Ph.D. degrees in electrical engineering from the California Institute of Technology, Pasadena, in 1999 and 2003, respectively.

During his studies, he spent one semester with the Department of Computer Science, the University of Edinburgh, Edinburgh, U.K., thanks to a fellowship of the Students Award Association of Scotland. He is currently a Postdoctoral Scholar at the University of California, Berkeley. His research interests include stochastic network models, wave propagation in random media, phase transitions, and distributed computing.

Dr. Franceschetti was a recipient of the 1999 UPE/IEEE Computer Society Award for academic excellence, the 2000 Caltech Walker von Brimer Foundation Award for outstanding research initiative, and the 2003 Caltech Wilts Prize for best thesis in electrical engineering.

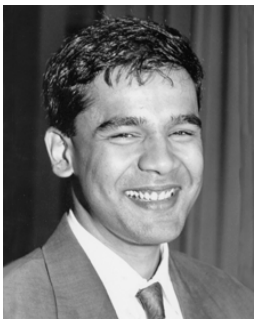

Kameshwar Poolla (M'02) received the B.Tech. degree from the Indian Institute of Technology, Bombay, in 1980, and the Ph.D. degree from the Center for Mathematical System Theory, University of Florida, Gainesville, in 1984, both in electrical engineering.

He has served on the Faculty of the Department of Electrical and Computer Engineering at the University of Illinois, Urbana-Champaign, from 1984 to 1991. Since then, he has been at the University of California, Berkeley, where he is now a Professor in the Departments of Mechanical Engineering and Electrical Engineering and Computer Sciences. He has also held visiting appointments at Honeywell Systems Research Center, Minneapolis, MN, McGill University, Montreal, QC, Canada, and the Massachusetts Institute of Technology, Cambridge, and has worked as a Field Engineer with Schlumberger AFR, Paris, France. He is a Co-founder of OnWafer Technologies, Dublin, CA, where he currently serves as Chief Scientist. His research interests include sensor networks, robust and adaptive control, system identification, semiconductor manufacturing, and mathematical biology.

Dr. Poolla was awarded the 1984 Outstanding Dissertation Award from the University of Florida, the 1988 NSF Presidential Young Investigator Award, the 1993 Hugo Schuck Best Paper Prize (jointly with Profs. Khargonekar, Tikku, Nagpal, and Krause), the 1994 Donald P. Eckman Award, a 1997 JSPS Fellowship, and the 1997 Distinguished Teaching Award from the University of California, Berkeley.

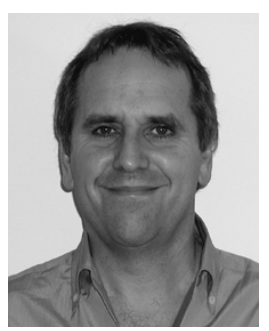

Michael I. Jordan (M'99-SM'99) received the Ph.D. from the University of California at San Diego, La Jolla, in 1986.

$\mathrm{He}$ is a Professor in the Department of Electrical Engineering and Computer Science and the Department of Statistics at the University of California, Berkeley. He was a Professor at the Massachusetts Institute of Technology, Cambridge, from 1988 to 1998. He has published over 200 research papers on topics in electrical engineering, computer science, statistics, and cognitive psychology. In recent years, he has focused on algorithms for probabilistic inference in graphical models and on applications of statistical learning theory to problems in bioinformatics, information retrieval, and signal processing.

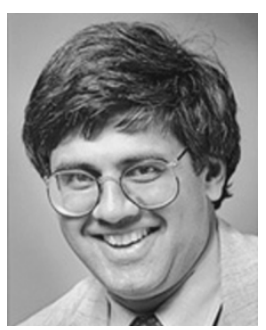

Shankar S. Sastry (S'79-M'80-SM'95-F'95) received the M.S. degree (honoris causa) from Harvard University, Cambridge, MA, in 1994, and the Ph.D. degree from the University of California, Berkeley, in 1981.

From 1980 to 1982, he was an Assistant Professor at the Massachusetts Institute of Technology, Cambridge. In 2000, he was Director of the Information Technology Office at the Defense Advanced Research Projects Agency (DARPA), Arlington, VA. He is currently the NEC Distinguished Professor of Electrical Engineering and Computer Sciences and Bioengineering and the Chairman of the Department of Electrical Engineering and Computer Sciences, the University of California, Berkeley. His research interests are embedded and autonomous software, computer vision, computation in novel substrates such as DNA, nonlinear and adaptive control, robotic telesurgery, control of hybrid systems, embedded systems, sensor networks, and biological motor control.

Dr. Sastry was elected into the National Academy of Engineering in 2000 for "pioneering contributions to the design of hybrid and embedded systems." He has served as an Associate Editor for the IEEE TRANSACTIONS ON Automatic CONTROL, the IEEE CONTROL SyStEMS MAGAZINE, and the IEEE TRANSACTIONS ON CIRCUITS AND SYSTEMS. 